\title{
Antimicrobial resistance surveillance in the South African public sector
}

\author{
Olga Perovic $^{a, b *}$, Husna Ismail ${ }^{a}$ and Erika Van Schalkwyk ${ }^{a}$ \\ ${ }^{a}$ Centre for Healthcare-Associated Infections (HAls), Antimicrobial Resistance (AMR) and Mycoses, National Institute for Communicable \\ Diseases, a division in the National Health Laboratory Service Johannesburg, South Africa \\ ${ }^{b}$ Department of Clinical Microbiology and Infectious Diseases, School of Pathology, University of Witwatersrand \\ Johannesburg, South Africa \\ ${ }^{*}$ Corresponding author, email: olgap@nicd.ac.za

Electronic surveillance for antimicrobial resistance was established in 2013 for public sector laboratories and released annually. This article reports susceptibility data on ESKAPE pathogens for 2016.

Keywords: antimicrobial resistance, ESKAPE organisms, surveillance

\section{Introduction}

Colonisation and infection due to multidrug-resistant (MDR) bacteria has become a significant public health concern with both clinical and economic consequences. ${ }^{1,2}$ Surveillance for antimicrobial resistance (AMR) is conducted not only to detect changes or variation in AMR either geographically or over time, but is a vital component of any antimicrobial stewardship programme. ${ }^{3}$ Integrated health data on bacterial AMR were obtained from an electronic database of antimicrobial susceptibility testing (AST) results generated by public health laboratories in South Africa. This report was designed to provide information on AMR rates in bacterial pathogens causing both community-associated and healthcare-associated infections and was prepared by the Centre for HAls, AMR and Mycoses (CHARM) and Surveillance Information Management Unit (SIMU) at the National Institute for Communicable Diseases (NICD) and Corporate Data Warehouse (CDW) at the National Health Laboratory Service (NHLS).

\section{Report objectives and scope}

1. To determine the number of cases for each of the following ESKAPE pathogens isolated from blood cultures in 2016: Enterococcus faecalis, Enterococcus faecium, Staphylococcus aureus, Klebsiella pneumoniae, Acinetobacter baumannii, Pseudomonas aeruginosa, Enterobacter cloacae, and Escherichia coli.

2. To compare AST patterns for each of the ESKAPE pathogens in 2016 with the previous year, 2015.

3. To describe the AST patterns for each of the ESKAPE pathogens by sentinel hospital in 2016.

4. To determine the number of laboratory-confirmed carbapenemase-producing Enterobacteriaceae (CPE) isolated from all specimen types in 2016.

\section{Methods}

\section{Data collection and analysis}

Data for this report were sourced from the NHLS, CDW. The CDW exists as a national repository for all laboratory tests performed from public sector hospitals in South Africa and contains archived data (demographic and laboratory) from the laboratory information system (LIS), TrakCare. These data were mapped as national, provincial, district and sentinel hospitals by the SIMU at NICD and are available in a dashboard from the NICD website, http://www.nicd.ac.za.

AMR surveillance in the public sector relies on submission of data from the NHLS laboratories that serve academic secondary and tertiary hospitals. ${ }^{4}$ Data containing routine AST results for the ESKAPE pathogens were extracted, from January 1, 2016 to December 31, 2016 for 16 sentinel hospitals across South Africa (Table 1). ${ }^{4}$

For the analysis of ESKAPE pathogens, AST results were interpreted in accordance with the Clinical and Laboratory Standards Institute (CLSI) 2016 guidelines and were categorised based on categorical data, susceptible (S) and non-susceptible including intermediate (I) and resistant (R). ${ }^{5}$ Due to site-specific differences in testing methodologies and data capture on the LIS, extensive cleaning and recording of data were necessary, which was done within the CDW (Table 2).

For the analysis of carbapenemase-producing Enterobacteriaceae (CPE), data were obtained from the Antimicrobial Resistance Laboratory (AMRL) at CHARM where carbapenemresistant isolates are referred for phenotypic characterisation, AST and molecular characterisation.

\section{Results}

For the purpose of this report, ESKAPE pathogens were categorised as Enterobacteriaceae (Klebsiella pneumoniae, Enterobacter cloacae, and Escherichia coli), non-fermentative Gram-negative bacteria (Acinetobacter baumannii and Pseudomonas aeruginosa) and Gram-positive bacteria (Enterococcus faecalis, Enterococcus faecium and Staphylococcus aureus).

\section{Enterobacteriaceae}

Of the 5265 lactose-fermenting bacteria, 53\% (2 783/5 265) were identified as Klebsiella pneumoniae, 35\% (1 850/5 265) were identified as Escherichia coli and 12\% (632/5265) were identified as Enterobacter cloacae. All three pathogens were reported from all 16 sentinel hospitals in South Africa. Some 21\% (1 095/5 265) of all three pathogens were reported from Chris Hani Baragwanath Hospital (Figure 1). 
Table 1: List of 16 sentinel hospitals participating in antimicrobial resistance surveillance

\begin{tabular}{|c|c|c|c|}
\hline Hospital name & Academic & $\begin{array}{l}\text { Number of } \\
\text { beds }\end{array}$ & Province \\
\hline $\begin{array}{l}\text { Charlotte Maxeke } \\
\text { Johannesburg Academic } \\
\text { Hospital }\end{array}$ & Yes & 1088 & Gauteng \\
\hline $\begin{array}{l}\text { Chris Hani Baragwanath } \\
\text { Hospital }\end{array}$ & Yes & 3200 & Gauteng \\
\hline $\begin{array}{l}\text { Dr George Mukhari } \\
\text { Hospital }\end{array}$ & Yes & 1200 & Gauteng \\
\hline Frere Hospital & No & 916 & Eastern Cape \\
\hline Grey's Hospital & Yes & 530 & KwaZulu-Natal \\
\hline Groote Schuur Hospital & Yes & 893 & Western Cape \\
\hline Helen Joseph Hospital & Yes & 700 & Gauteng \\
\hline $\begin{array}{l}\text { Inkosi Albert Luthuli } \\
\text { Central Hospital }\end{array}$ & Yes & 846 & KwaZulu-Natal \\
\hline $\begin{array}{l}\text { King Edward VIII } \\
\text { Hospital }\end{array}$ & Yes & 922 & KwaZulu-Natal \\
\hline Livingstone Hospital & Yes & 616 & Eastern Cape \\
\hline $\begin{array}{l}\text { Mahatma Gandhi } \\
\text { Hospital }\end{array}$ & No & 350 & KwaZulu-Natal \\
\hline $\begin{array}{l}\text { Nelson Mandela } \\
\text { Academic Hospital/ } \\
\text { Mthatha Tertiary }\end{array}$ & Yes & 520 & Eastern Cape \\
\hline RK Khan Hospital & No & 543 & KwaZulu-Natal \\
\hline $\begin{array}{l}\text { Steve Biko Academic } \\
\text { Hospital }\end{array}$ & Yes & 832 & Gauteng \\
\hline Tygerberg Hospital & Yes & 1310 & Western Cape \\
\hline Universitas Hospital & Yes & 650 & Free State \\
\hline
\end{tabular}

Of the panel of antimicrobial agents that were tested, more than $65 \%$ of Klebsiella pneumoniae isolates were non-susceptible to third and fourth generation cephalosporins, which is indicative of extended-spectrum beta-lactamase (ESBL) production. In total, 36\% (952/2 642) of Klebsiella pneumoniae isolates were non-susceptible to ciprofloxacin, 44\% (1 183/2 686) of isolates were non-susceptible to piperacillin/tazobactam and 59\% (1 $568 / 2$ 676) were non-susceptible to gentamicin (Table 3 ). In comparison with 2015, Klebsiella pneumoniae isolates
Table 2: Antimicrobial susceptibility testing methods performed at the 16 sentinel hospitals

\begin{tabular}{|l|c|c|c|}
\hline $\begin{array}{l}\text { NHLS laboratories at } \\
\text { public sector hospitals }\end{array}$ & MicroScan & Vitek 2 & $\begin{array}{c}\text { Disk } \\
\text { diffusion } \\
\text { method }\end{array}$ \\
\hline $\begin{array}{l}\text { Charlotte Maxeke } \\
\text { Johannesburg Academic } \\
\text { Hospital }\end{array}$ & $\sqrt{ }$ & $\sqrt{ }$ & $\sqrt{ }$ \\
\hline $\begin{array}{l}\text { Chris Hani Baragwanath } \\
\text { Hospital }\end{array}$ & $\sqrt{ }$ & $\sqrt{ }$ \\
\hline Dr George Mukhari Hospital & & $\sqrt{ }$ & \\
\hline Frere Hospital & & $\sqrt{ }$ & \\
\hline $\begin{array}{l}\text { Grey's Hospital/Northdale } \\
\text { Laboratory }\end{array}$ & & $\sqrt{ }$ & \\
\hline Groote Schuur Hospital & & $\sqrt{ }$ & $\sqrt{ }$ \\
\hline Helen Joseph Hospital & & $\sqrt{ }$ & \\
\hline Inkosi Albert Luthuli Central & & $\sqrt{ }$ & \\
\hline Hospital & & $\sqrt{ }$ & \\
\hline King Edward VIII Hospital & & $\sqrt{ }$ & \\
\hline Livingstone Hospital & & & $\sqrt{ }$ \\
\hline Mahatma Gandhi Hospital & & $\sqrt{ }$ & \\
\hline $\begin{array}{l}\text { Nelson Mandela Academic } \\
\text { Hospital/Mthatha Tertiary }\end{array}$ & & $\sqrt{ }$ & \\
\hline RK Khan Hospital & & & \\
\hline Steve Biko Academic & & & \\
\hline Hospital & & & \\
\hline Tygerberg Hospital & & & \\
\hline Universitas Hospital & & \\
\hline
\end{tabular}

demonstrated higher susceptibility to cefepime $(p=0.65)$, piperacillin/tazobactam $(p=0.26)$ and gentamicin in 2016. Although a higher susceptibility was observed for cefepime and piperacillin/ tazobactam in 2016, this was not statistically significant. Overall, antimicrobial susceptibility to carbapenems remained constant over the two-year period (Figure 2). However, high proportions of Klebsiella pneumoniae isolates reported from King Edward VIII Hospital Grey's Hospital, Frere Hospital and Nelson Mandela Academic Hospital/Mthatha Tertiary were shown to display reduced susceptibility to cephalosporins (Table 4).

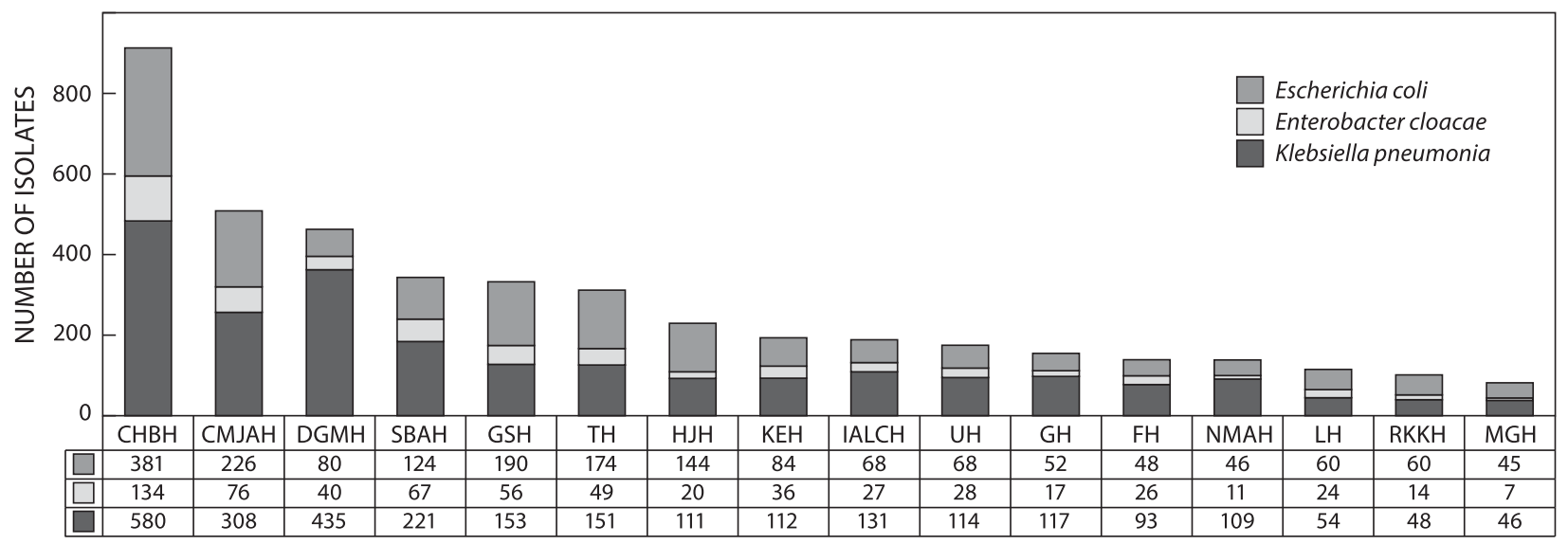

Figure 1: Number of Enterobacteriaceae: Klebsiella pneumoniae ( $n=2783)$, Escherichia coli $(n=1850)$ and Enterobacter cloacae $(n=632)$ reported from 16 sentinel hospitals across South Africa, January 1, 2016 to December 31, 2016. Abbreviations: Chris Hani Baragwanath Hospital (CHBH), Charlotte Maxeke Johannesburg Academic Hospital (CMJAH), Dr George Mukhari Hospital (DGMH), Steve Biko Academic Hospital (SBAH), Groote Schuur Hospital (GSH), Tygerberg Hospital (TH), Helen Joseph Hospital (HJH), King Edward VIII Hospital (KEH), Inkosi Albert Luthuli Central Hospital (IALCH), Universitas Hospital (UH), Grey's Hospital (GH), Frere Hospital (FH), Nelson Mandela Academic Hospital/Mthatha Tertiary (NMAH), Livingstone Hospital (LH), RK Khan Hospital (RKKH) and Mahatma Gandhi Hospital (MGH), number of isolates (n). 
Table 3: Antimicrobial susceptibility patterns of Enterobacteriaceae isolated from blood cultures reported from 16 sentinel hospitals across South Africa, January 1, 2016 to December 31, 2016

\begin{tabular}{|c|c|c|c|c|c|c|c|c|}
\hline \multirow{3}{*}{ Antimicrobial agent } & \multicolumn{4}{|c|}{ Klebsiella pneumoniae } & \multicolumn{4}{|c|}{ Escherichia coli } \\
\hline & \multicolumn{2}{|c|}{ Non-susceptible } & \multicolumn{2}{|c|}{ Susceptible } & \multicolumn{2}{|c|}{ Non-susceptible } & \multicolumn{2}{|c|}{ Susceptible } \\
\hline & $n$ & $\%$ & $n$ & $\%$ & $n$ & $\%$ & $n$ & $\%$ \\
\hline Amikacin & 442 & 16.4 & 2251 & 83.6 & 160 & 8.8 & 1651 & 91.2 \\
\hline Amoxicillin-clavulanic acid & 1785 & 66.3 & 909 & 33.7 & 708 & 39.2 & 1096 & 60.8 \\
\hline Ampicillin/amoxicillin & - & - & - & - & 1539 & 86.3 & 244 & 13.7 \\
\hline Cefepime & 1748 & 65.0 & 941 & 35.0 & 470 & 26.5 & 1305 & 73.5 \\
\hline Cefotaxime/ceftriaxone & 1779 & 66.0 & 916 & 34.0 & 500 & 27.8 & 1297 & 72.2 \\
\hline Ceftazidime & 1768 & 65.7 & 921 & 34.3 & 483 & 26.8 & 1317 & 73.2 \\
\hline Ciprofloxacin & 952 & 36.0 & 1690 & 64.0 & 530 & 30.1 & 1230 & 69.9 \\
\hline Ertapenem & 137 & 5.2 & 2476 & 94.8 & 23 & 1.3 & 1754 & 98.7 \\
\hline Gentamicin & 1568 & 58.6 & 1108 & 41.4 & 348 & 19.5 & 1441 & 80.5 \\
\hline Imipenem & 168 & 6.2 & 2541 & 93.8 & 14 & 0.8 & 1797 & 99.2 \\
\hline Meropenem & 178 & 6.6 & 2535 & 93.4 & 15 & 0.8 & 1797 & 99.2 \\
\hline Piperacillin/tazobactam & 1183 & 44.0 & 1503 & 56.0 & 257 & 14.5 & 1513 & 85.5 \\
\hline
\end{tabular}

Notes: number of isolates $(n)$, percentage (\%), not reported (-).

Colistin was not reported as no reference method was applied at routine laboratories.
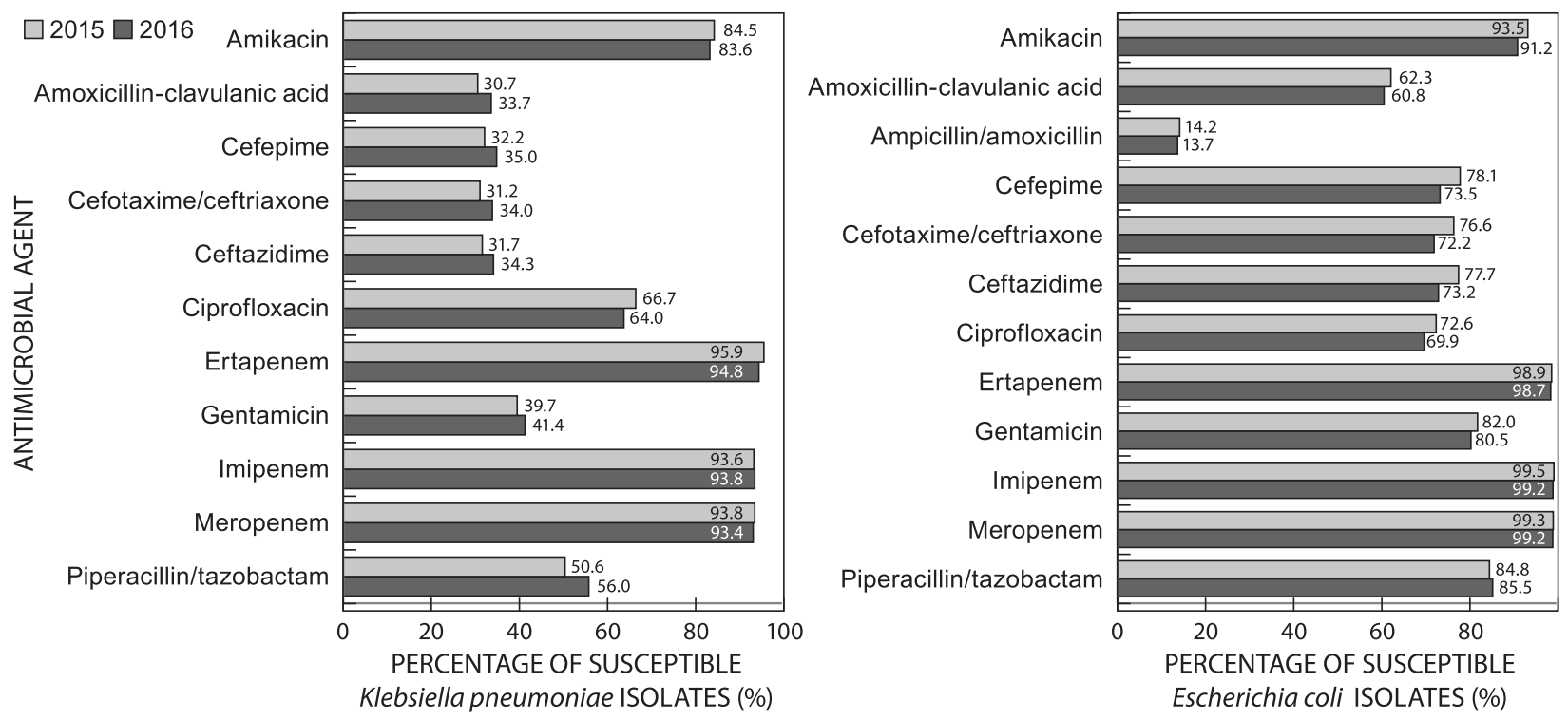

Figure 2: Percentage of susceptible Klebsiella pneumoniae and Escherichia coli isolates, 2015 to 2016.

Less than $30 \%$ of Escherichia coli isolates were non-susceptible to third and fourth generation cephalosporins and 30\% (530/1760) of isolates were non-susceptible to ciprofloxacin (see Table 3 ). In comparison with 2015, Escherichia coli isolates showed reduced susceptibility in almost all antimicrobial agents (Figure 2). Overall, high proportions of Escherichia coli isolates were shown to be susceptible to carbapenems across all 16 sentinel hospitals (Table 5).

Antimicrobial susceptibility patterns for Enterobacter cloacae were not reported as data were not available during the preparation of this report.

\section{Non-fermentative gram-negative bacteria}

Of the 2318 non-fermentative Gram-negative bacteria, 71\% (1 637/2 318) were identified as Acinetobacter baumannii and 29\% (681/2 318) were identified as Pseudomonas aeruginosa. Both pathogens were reported from all 16 sentinel hospitals in South Africa. Approximately 32\% (738/2 318) of both pathogens were reported from Chris Hani Baragwanath Hospital (Figure 3).

Of the panel of antimicrobial agents that were tested, more than $80 \%$ of Acinetobacter baumannii isolates were non-susceptible to imipenem and meropenem, while $72 \%$ (1 140/1 583) and 60\% (791/1 320) were non-susceptible to gentamicin and amikacin (Table 6). In comparison to 2015, isolates non-susceptible to gentamicin and amikacin increased but, susceptibility to carbapenems and tigecycline remained constant (Figure 4). A high proportion of Acinetobacter baumannii isolates reported from Chris Hani Baragwanath Hospital, Charlotte Maxeke Johannesburg Academic Hospital, Dr George Mukhari Hospital, Helen Joseph Hospital, Inkosi Albert Luthuli Central Hospital, King Edward VIII Hospital, Steve Biko Academic Hospital and Universitas Hospital showed reduced susceptibility to carbapenems (Table 7). 
Table 4: Number and percentage of susceptible Klebsiella pneumoniae isolates per antimicrobial agent from 16 sentinel hospitals across South Africa, January 1, 2016 to December 31 , 2016

\begin{tabular}{|c|c|c|c|c|c|c|c|c|c|c|c|c|c|c|c|c|}
\hline $\begin{array}{l}\text { Antimicrobial } \\
\text { agent }\end{array}$ & CHBH & CMJAH & DGMH & SBAH & GSH & TH & $\mathrm{HJH}$ & $\mathrm{KEH}^{*}$ & IALCH* $^{*}$ & UH & $\mathrm{GH}^{*}$ & $\mathrm{FH}$ & NMAH & LH & $\mathrm{RKKH}^{*}$ & $\mathrm{MGH}^{*}$ \\
\hline & $n$ & $n$ & $n$ & $n$ & $n$ & $n$ & $n$ & $n$ & $n$ & $n$ & $n$ & $n$ & $n$ & $n$ & $n$ & $n$ \\
\hline & $\%$ & $\%$ & $\%$ & $\%$ & $\%$ & $\%$ & $\%$ & $\%$ & $\%$ & $\%$ & $\%$ & $\%$ & $\%$ & $\%$ & $\%$ & $\%$ \\
\hline \multirow[t]{2}{*}{ Amikacin } & 560 & 288 & 416 & 220 & 151 & 149 & 106 & 106 & 130 & 112 & 113 & 91 & 106 & 54 & 47 & 44 \\
\hline & 94.8 & 93.8 & 89.2 & 64.1 & 94.7 & 81.2 & 98.1 & 60.4 & 67.7 & 87.5 & 88.5 & 64.8 & 45.3 & 87.0 & 76.6 & 68.2 \\
\hline \multirow{2}{*}{$\begin{array}{l}\text { Amoxicillin- } \\
\text { clavulanic acid }\end{array}$} & 556 & 292 & 413 & 221 & 152 & 150 & 107 & 109 & 128 & 111 & 113 & 90 & 106 & 54 & 48 & 44 \\
\hline & 27.0 & 32.9 & 56.4 & 29.9 & 38.2 & 38.7 & 36.4 & 21.1 & 27.3 & 33.3 & 15.9 & 15.6 & 20.8 & 40.7 & 43.8 & 38.6 \\
\hline \multirow[t]{2}{*}{ Cefepime } & 566 & 297 & 413 & 221 & 152 & 150 & 110 & 100 & 120 & 111 & 111 & 90 & 105 & 53 & 45 & 45 \\
\hline & 32.5 & 38.0 & 58.1 & 33.0 & 41.1 & 40.0 & 44.5 & 15.0 & 25.0 & 35.1 & 14.4 & 13.3 & 4.8 & 32.1 & 31.1 & 24.4 \\
\hline \multirow{2}{*}{$\begin{array}{l}\text { Cefotaxime/ } \\
\text { ceftriaxone }\end{array}$} & 556 & 296 & 414 & 221 & 150 & 151 & 107 & 104 & 129 & 111 & 113 & 92 & 105 & 54 & 47 & 45 \\
\hline & 31.7 & 35.8 & 56.3 & 33.0 & 41.3 & 39.7 & 40.2 & 15.4 & 24.8 & 35.1 & 14.2 & 12.0 & 5.7 & 31.5 & 29.8 & 26.7 \\
\hline \multirow[t]{2}{*}{ Ceftazidime } & 563 & 290 & 412 & 221 & 149 & 150 & 109 & 105 & 128 & 111 & 113 & 89 & 105 & 54 & 46 & 44 \\
\hline & 32.5 & 36.9 & 55.8 & 33.0 & 41.6 & 40.0 & 39.4 & 16.2 & 25.0 & 35.1 & 14.2 & 12.4 & 3.8 & 33.3 & 30.4 & 27.3 \\
\hline \multirow[t]{2}{*}{ Ciprofloxacin } & 553 & 244 & 416 & 221 & 151 & 151 & 107 & 107 & 129 & 111 & 112 & 90 & 105 & 54 & 46 & 45 \\
\hline & 56.6 & 65.2 & 79.6 & 55.2 & 72.8 & 74.2 & 50.5 & 43.0 & 57.4 & 61.3 & 63.4 & 62.2 & 85.7 & 59.3 & 56.5 & 57.8 \\
\hline \multirow[t]{2}{*}{ Ertapenem } & 559 & 287 & 426 & 205 & 151 & 150 & 110 & 79 & 103 & 112 & 112 & 88 & 104 & 50 & 42 & 35 \\
\hline & 86.8 & 93.7 & 99.1 & 92.2 & 99.3 & 96.7 & 90.0 & 97.5 & 100.0 & 98.2 & 97.3 & 100.0 & 99.0 & 100.0 & 100.0 & 100.0 \\
\hline \multirow[t]{2}{*}{ Gentamicin } & 550 & 289 & 415 & 221 & 151 & 151 & 108 & 105 & 120 & 111 & 113 & 91 & 105 & 54 & 47 & 45 \\
\hline & 32.2 & 47.8 & 62.7 & 43.4 & 49.7 & 53.0 & 55.6 & 27.6 & 27.5 & 37.8 & 18.6 & 25.3 & 7.6 & 55.6 & 40.4 & 37.8 \\
\hline \multirow[t]{2}{*}{ Imipenem } & 564 & 298 & 422 & 218 & 148 & 151 & 110 & 109 & 130 & 111 & 112 & 87 & 105 & 53 & 46 & 45 \\
\hline & 91.5 & 92.3 & 99.8 & 86.2 & 99.3 & 98.0 & 96.4 & 79.8 & 86.9 & 99.1 & 95.5 & 100.0 & 100.0 & 100.0 & 93.5 & 77.8 \\
\hline \multirow[t]{2}{*}{ Meropenem } & 558 & 298 & 426 & 220 & 153 & 149 & 109 & 105 & 130 & 112 & 113 & 90 & 106 & 52 & 46 & 46 \\
\hline & 91.9 & 92.6 & 99.3 & 85.0 & 99.3 & 97.3 & 96.3 & 80.0 & 86.2 & 97.3 & 94.7 & 98.9 & 97.2 & 100.0 & 91.3 & 78.3 \\
\hline \multirow{2}{*}{$\begin{array}{l}\text { Piperacillin/ } \\
\text { tazobactam }\end{array}$} & 564 & 296 & 423 & 220 & 152 & 151 & 109 & 108 & 130 & 110 & 111 & 63 & 103 & 54 & 47 & 45 \\
\hline & 57.8 & 60.8 & 66.7 & 27.3 & 75.7 & 78.8 & 49.5 & 45.4 & 45.4 & 33.6 & 43.2 & 74.6 & 34.0 & 50.0 & 78.7 & 62.2 \\
\hline
\end{tabular}

Note: *AST patterns for carbapenems varied for sentinel hospitals located in KwaZulu-Natal: KEH, IALCH, GH, RKKH and MGH. 
Table 5: Number and percentage of susceptible Escherichia coli isolates per antimicrobial agent from 16 sentinel hospitals across South Africa, January 1, 2016 to December 31,2016

\begin{tabular}{|c|c|c|c|c|c|c|c|c|c|c|c|c|c|c|c|c|}
\hline $\begin{array}{l}\text { Antimicrobial } \\
\text { agent }\end{array}$ & CHBH & CMJAH & DGMH & SBAH & GSH & TH & HJH & KEH & IALCH & UH & GH & FH & NMAH & LH & RKKH & MGH \\
\hline & $n$ & $n$ & $n$ & $n$ & $n$ & $n$ & $n$ & $n$ & $n$ & $n$ & $n$ & $n$ & $n$ & $n$ & $n$ & $n$ \\
\hline & $\%$ & $\%$ & $\%$ & $\%$ & $\%$ & $\%$ & $\%$ & $\%$ & $\%$ & $\%$ & $\%$ & $\%$ & $\%$ & $\%$ & $\%$ & $\%$ \\
\hline \multirow{2}{*}{ Amikacin } & 373 & 221 & 79 & 124 & 186 & 171 & 138 & 83 & 68 & 67 & 51 & 47 & 43 & 59 & 57 & 44 \\
\hline & 98.7 & 98.2 & 81.0 & 74.2 & 98.9 & 91.8 & 99.3 & 71.1 & 69.1 & 89.6 & 84.3 & 91.5 & 86.0 & 86.4 & 89.5 & 93.2 \\
\hline \multirow{2}{*}{$\begin{array}{l}\text { Amoxicillin- } \\
\text { clavulanic acid }\end{array}$} & 372 & 220 & 78 & 124 & 185 & 173 & 137 & 81 & 67 & 66 & 52 & 46 & 45 & 58 & 57 & 43 \\
\hline & 52.4 & 68.2 & 56.4 & 54.8 & 73.0 & 71.1 & 63.5 & 42.0 & 47.8 & 74.2 & 40.4 & 82.6 & 46.7 & 67.2 & 56.1 & 65.1 \\
\hline \multirow{2}{*}{$\begin{array}{l}\text { Ampicillin/ } \\
\text { amoxicillin }\end{array}$} & 363 & 215 & 74 & 123 & 185 & 171 & 134 & 81 & 67 & 67 & 52 & 48 & 43 & 59 & 57 & 44 \\
\hline & 4.4 & 16.7 & 10.8 & 21.1 & 22.7 & 21.6 & 10.4 & 4.9 & 7.5 & 22.4 & 3.8 & 18.8 & 14.0 & 23.7 & 14.0 & 4.5 \\
\hline \multirow{2}{*}{ Cefepime } & 368 & 221 & 75 & 123 & 185 & 172 & 143 & 79 & 56 & 67 & 52 & 47 & 43 & 59 & 52 & 33 \\
\hline & 74.2 & 89.6 & 58.7 & 71.5 & 73.5 & 77.9 & 83.2 & 48.1 & 48.2 & 91.0 & 55.8 & 83.0 & 46.5 & 78.0 & 63.5 & 60.6 \\
\hline \multirow{2}{*}{$\begin{array}{l}\text { Cefotaxime/ } \\
\text { ceftriaxone }\end{array}$} & 369 & 218 & 78 & 124 & 185 & 171 & 141 & 82 & 66 & 66 & 52 & 45 & 40 & 59 & 57 & 44 \\
\hline & 72.9 & 81.7 & 59.0 & 71.8 & 74.1 & 78.4 & 80.9 & 48.8 & 51.5 & 90.9 & 57.7 & 82.2 & 50.0 & 78.0 & 63.2 & 61.4 \\
\hline \multirow{2}{*}{ Ceftazidime } & 365 & 221 & 76 & 123 & 187 & 172 & 142 & 81 & 65 & 67 & 52 & 46 & 44 & 59 & 56 & 44 \\
\hline & 73.7 & 87.8 & 57.9 & 71.5 & 73.3 & 77.9 & 82.4 & 51.9 & 50.8 & 91.0 & 57.7 & 84.8 & 47.7 & 78.0 & 62.5 & 61.4 \\
\hline \multirow[t]{2}{*}{ Ciprofloxacin } & 364 & 185 & 77 & 122 & 184 & 169 & 141 & 82 & 67 & 67 & 51 & 47 & 44 & 58 & 57 & 45 \\
\hline & 70.9 & 79.5 & 61.0 & 69.7 & 65.8 & 72.2 & 80.1 & 54.9 & 47.8 & 83.6 & 52.9 & 78.7 & 75.0 & 79.3 & 64.9 & 53.3 \\
\hline \multirow[t]{2}{*}{ Ertapenem } & 375 & 222 & 77 & 122 & 184 & 173 & 140 & 77 & 58 & 66 & 52 & 45 & 42 & 54 & 51 & 34 \\
\hline & 94.9 & 99.5 & 100.0 & 99.2 & 100.0 & 100.0 & 99.3 & 98.7 & 100.0 & 100.0 & 100.0 & 100.0 & 100.0 & 100.0 & 100.0 & 100.0 \\
\hline \multirow[t]{2}{*}{ Gentamicin } & 367 & 220 & 77 & 123 & 185 & 170 & 141 & 81 & 59 & 66 & 52 & 47 & 43 & 59 & 56 & 43 \\
\hline & 79.6 & 83.6 & 76.6 & 86.2 & 81.1 & 86.5 & 83.7 & 69.1 & 61 & 86.4 & 69.2 & 89.4 & 81.4 & 93.2 & 76.8 & 58.1 \\
\hline \multirow[t]{2}{*}{ Imipenem } & 376 & 224 & 78 & 124 & 186 & 173 & 142 & 83 & 67 & 66 & 52 & 47 & 41 & 59 & 53 & \\
\hline & 97.9 & 99.1 & 100.0 & 98.4 & 100.0 & 100.0 & 98.6 & 100.0 & 100.0 & 100.0 & 100.0 & 100.0 & 100.0 & 100.0 & 100.0 & 100.0 \\
\hline \multirow[t]{2}{*}{ Meropenem } & 371 & 223 & 78 & 123 & 185 & 172 & 143 & 82 & 67 & 67 & 52 & 47 & 42 & 59 & 58 & 40 \\
\hline & 97.6 & 99.1 & 100.0 & 98.4 & 100.0 & 100.0 & 98.6 & 100.0 & 100.0 & 100.0 & 100.0 & 100.0 & 100.0 & 100.0 & 100.0 & 100.0 \\
\hline \multirow{2}{*}{$\begin{array}{l}\text { Piperacillin/ } \\
\text { tazobactam }\end{array}$} & 369 & 220 & 79 & 124 & 187 & 173 & 141 & 82 & 68 & 67 & 52 & - & 43 & 59 & 58 & 45 \\
\hline & 87.0 & 86.4 & 77.2 & 62.9 & 93.0 & 90.8 & 85.1 & 82.9 & 88.2 & 76.1 & 78.8 & - & 81.4 & 94.9 & 94.8 & 95.6 \\
\hline
\end{tabular}

Note: Data were omitted for those sentinel hospitals that tested fewer than 30 ESKAPE pathogens for a particular antimicrobial agent. 


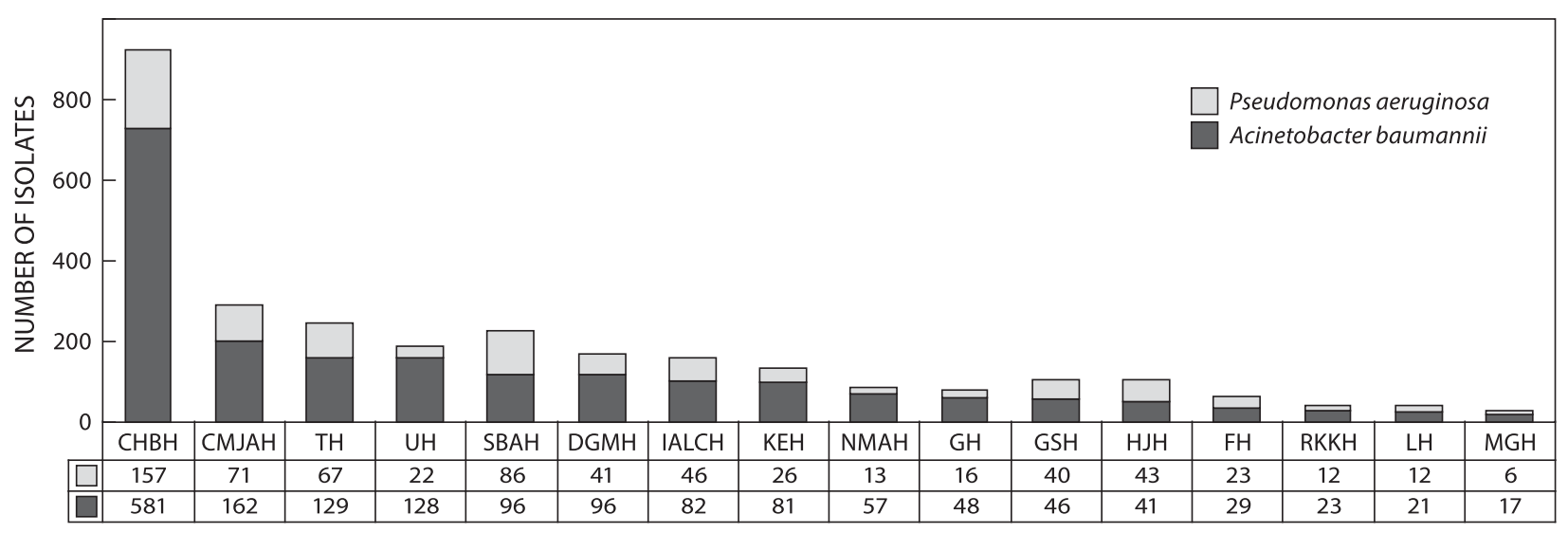

Figure 3: Number of non-fermenters: Acinetobacter baumannii $(n=1637)$ and Pseudomonas aeruginosa $(n=681)$ reported from 16 sentinel hospitals across South Africa, January 1, 2016 to December 31, 2016.

Table 6: Antimicrobial susceptibility patterns of non-fermenters isolated from blood cultures reported from 16 sentinel hospitals across South Africa, January 1, 2016 to December 31, 2016

\begin{tabular}{|l|c|c|c|c|c|c|c|c|}
\hline \multirow{2}{*}{ Antimicrobial agent } & \multicolumn{4}{|c|}{ Acinetobacter baumannii } & \multicolumn{4}{c|}{ Pseudomonas aeruginosa } \\
\cline { 2 - 11 } & \multicolumn{2}{|c|}{ Non-susceptible } & \multicolumn{2}{|c|}{ Susceptible } & \multicolumn{2}{c|}{ Non-susceptible } & \multicolumn{3}{c|}{ Susceptible } \\
\hline & $n$ & $\%$ & $n$ & $\%$ & $n$ & $\%$ & $n$ & $\%$ \\
\hline Amikacin & 791 & 59.9 & 529 & 40.1 & - & - & - & - \\
\hline Gentamicin & 1140 & 72.0 & 443 & 28.0 & - & - & - & - \\
\hline Imipenem & 1294 & 81.0 & 304 & 19.0 & 172 & 26.1 & 488 & 73.9 \\
\hline Meropenem & 1290 & 81.3 & 296 & 18.7 & 164 & 24.8 & 498 & 75.2 \\
\hline Minocycline & 21 & 87.5 & 3 & 12.5 & - & - & - & - \\
\hline Tigecycline & 53 & 7.5 & 652 & 92.5 & - & - & - & - \\
\hline Cefepime & - & - & - & - & 139 & 21.75 & 500 & 78.2 \\
\hline Ceftazidime & - & - & - & - & 134 & 20.12 & 532 & 79.9 \\
\hline Piperacillin/tazobactam & - & - & - & - & 151 & 23.45 & 493 & 76.6 \\
\hline
\end{tabular}

Notes: number of isolates (n), percentage (\%), not reported (-).
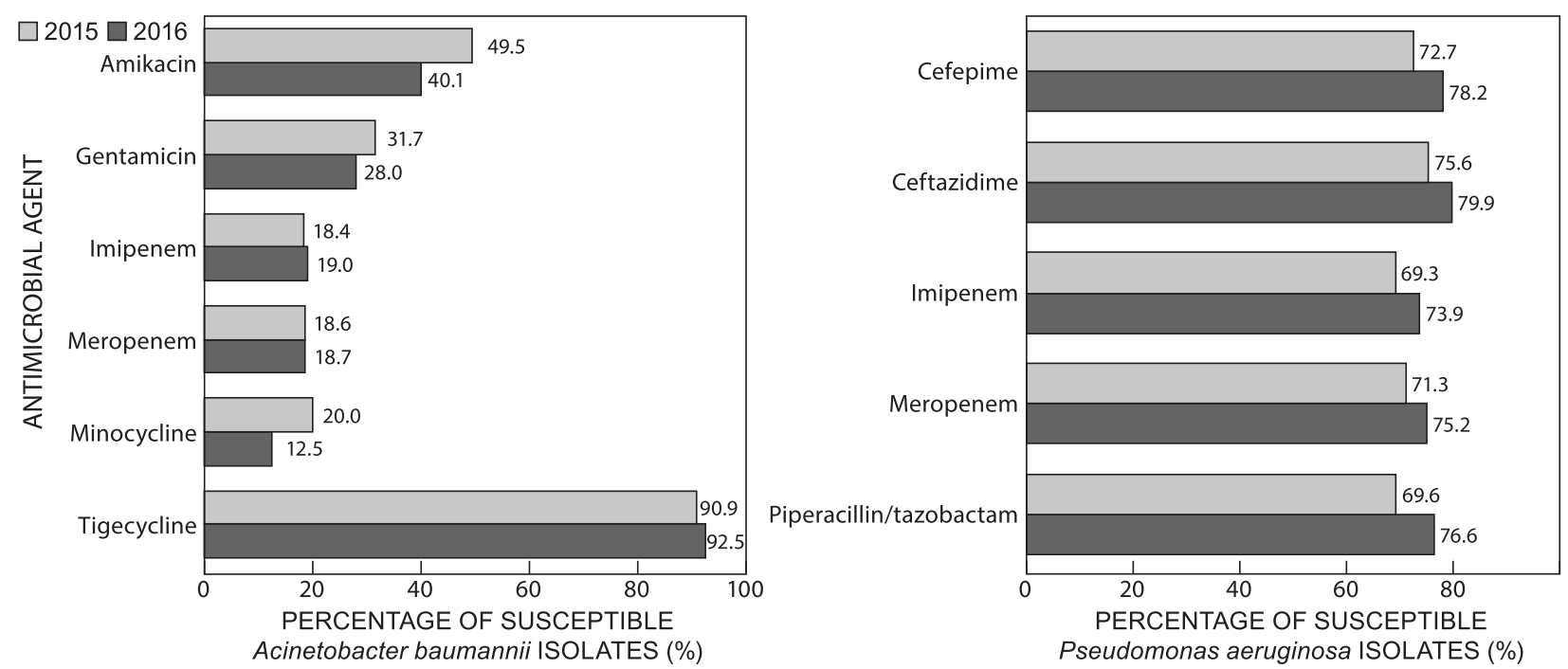

Figure 4: Percentage of susceptible Acinetobacter baumannii and Pseudomonas aeruginosa isolates, 2015 to 2016.

Approximately $80 \%$ and $75 \%$ of Pseudomonas aeruginosa isolates were susceptible to cephalosporins and carbapenems (Table 6). Antimicrobial susceptibility to imipenem ( $p=0.21)$, cefepime $(p=0.57)$ and piperacillin/tazobactam $(p=0.39)$ increased in Pseudomonas aeruginosa; however, this was not statistically significant over the two-year period (Figure 4). Almost $50 \%$ of
Pseudomonas aeruginosa isolates reported from Tygerberg Hospital showed reduced susceptibility to carbapenems (Table 8).

\section{Gram-positive bacteria}

Of the 3369 Gram-positive bacteria, 20\% (785/3 369) were identified as Enterococcus faecalis, $21 \%$ (846/3 369) were identified as 
Table 7: Number and percentage of susceptible Acinetobacter baumannii isolates per antimicrobial agent from 16 sentinel hospitals across South Africa, January 1, 2016 to December 31 , 2016

\begin{tabular}{|c|c|c|c|c|c|c|c|c|c|c|c|c|c|c|c|c|}
\hline $\begin{array}{l}\text { Antimicrobial } \\
\text { agent }\end{array}$ & CHBH & CMJAH & DGMH & SBAH & GSH & TH & $\mathrm{HJH}$ & KEH & IALCH & UH & GH & FH & NMAH & LH & RKKH & MGH \\
\hline & $n$ & $n$ & $n$ & $n$ & $n$ & $n$ & $n$ & $n$ & $n$ & $n$ & $n$ & $n$ & $n$ & $n$ & $n$ & $n$ \\
\hline & $\%$ & $\%$ & $\%$ & $\%$ & $\%$ & $\%$ & $\%$ & $\%$ & $\%$ & $\%$ & $\%$ & $\%$ & $\%$ & $\%$ & $\%$ & $\%$ \\
\hline \multirow[t]{2}{*}{ Amikacin } & 561 & 110 & 91 & - & 44 & 119 & 36 & 47 & 68 & 120 & 43 & - & - & - & - & - \\
\hline & 32.6 & 43.6 & 34.1 & - & 70.5 & 41.2 & 41.7 & 70.2 & 72.1 & 20.8 & 39.5 & - & - & - & - & - \\
\hline \multirow[t]{2}{*}{ Gentamicin } & 557 & 155 & 96 & 96 & 44 & 125 & 40 & 77 & 75 & 125 & 48 & - & 56 & - & - & - \\
\hline & 16.5 & 35.5 & 21.9 & 21.9 & 75.0 & 44.8 & 37.5 & 41.6 & 42.7 & 10.4 & 43.8 & - & 23.2 & - & - & - \\
\hline \multirow[t]{2}{*}{ Imipenem } & 567 & 157 & 94 & 96 & 46 & 125 & 40 & 77 & 78 & 125 & 48 & - & 56 & - & - & - \\
\hline & 6.7 & 12.1 & 24.5 & 17.7 & 58.7 & 26.4 & 12.5 & 33.8 & 33.3 & 9.6 & 20.8 & - & 46.4 & - & - & - \\
\hline \multirow[t]{2}{*}{ Meropenem } & 558 & 157 & 94 & 96 & 43 & 126 & 38 & 77 & 78 & 126 & 47 & - & 57 & - & - & - \\
\hline & 6.1 & 12.1 & 25.5 & 17.7 & 58.1 & 25.4 & 15.8 & 32.5 & 33.3 & 9.5 & 21.3 & - & 47.4 & - & - & - \\
\hline \multirow[t]{2}{*}{ Tigecycline } & - & 136 & 86 & 95 & 44 & - & - & 76 & 75 & - & 47 & - & 54 & - & - & - \\
\hline & - & 94.9 & 95.3 & 100.0 & 95.5 & - & - & 93.4 & 84.0 & - & 87.2 & - & 96.3 & - & - & - \\
\hline
\end{tabular}

Note: Data were omitted for those sentinel hospitals that tested fewer than 30 ESKAPE pathogens for a particular antimicrobial agent.

Table 8: Number and percentage of susceptible Pseudomonas aeruginosa isolates per antimicrobial agent from 16 sentinel hospitals across South Africa, January 1, 2016 to December 31,2016

\begin{tabular}{|c|c|c|c|c|c|c|c|c|c|c|c|c|c|c|c|c|}
\hline $\begin{array}{l}\text { Antimicrobial } \\
\text { agent }\end{array}$ & CHBH & СMJAH & DGMH & SBAH & GSH & TH & HJH & KEH & IALCH & UH & GH & FH & NMAH & LH & RKKH & MGH \\
\hline & $n$ & $n$ & $n$ & $n$ & $n$ & $n$ & $n$ & $n$ & $n$ & $n$ & $n$ & $n$ & $n$ & $n$ & $n$ & $n$ \\
\hline & $\%$ & $\%$ & $\%$ & $\%$ & $\%$ & $\%$ & $\%$ & $\%$ & $\%$ & $\%$ & $\%$ & $\%$ & $\%$ & $\%$ & $\%$ & $\%$ \\
\hline \multirow[t]{2}{*}{ Cefepime } & 154 & 69 & 39 & 88 & 38 & 65 & 42 & - & 34 & - & - & - & - & - & - & - \\
\hline & 87.0 & 73.9 & 87.2 & 76.1 & 78.9 & 63.1 & 95.2 & - & 58.8 & - & - & - & - & - & - & - \\
\hline \multirow[t]{2}{*}{ Ceftazidime } & 153 & 69.0 & 40.0 & 88 & 37 & 65 & 43 & - & 44 & - & - & - & - & - & - & - \\
\hline & 86.3 & 75.4 & 92.5 & 76.1 & 81.1 & 66.2 & 93.0 & - & 72.7 & - & - & - & - & - & - & - \\
\hline \multirow[t]{2}{*}{ Imipenem } & 153 & 70 & 38 & 88 & 39 & 64 & 42 & - & 45 & - & - & - & - & - & - & - \\
\hline & 70.6 & 72.9 & 92.1 & 65.9 & 74.4 & 48.4 & 88.1 & - & 77.8 & - & - & - & - & - & - & - \\
\hline \multirow[t]{2}{*}{ Meropenem } & 152 & 70 & 38 & 88 & 38.0 & 65 & 41 & - & 45 & - & - & - & - & - & - & - \\
\hline & 70.4 & 74.3 & 89.5 & 67.0 & 76.3 & 52.3 & 92.7 & - & 77.8 & - & - & - & - & - & - & - \\
\hline \multirow[t]{2}{*}{$\begin{array}{l}\text { Piperacillin/ } \\
\text { tazobactam }\end{array}$} & 154 & 67 & 40 & 84 & 38 & 62 & 42 & - & 45 & - & - & - & - & - & - & - \\
\hline & 79.2 & 71.6 & 87.5 & 76.2 & 76.3 & 79.0 & 85.7 & - & 71.1 & - & - & - & - & - & - & - \\
\hline
\end{tabular}

Note: Data were omitted for those sentinel hospitals that tested fewer than 30 ESKAPE pathogens for a particular antimicrobial agent. 


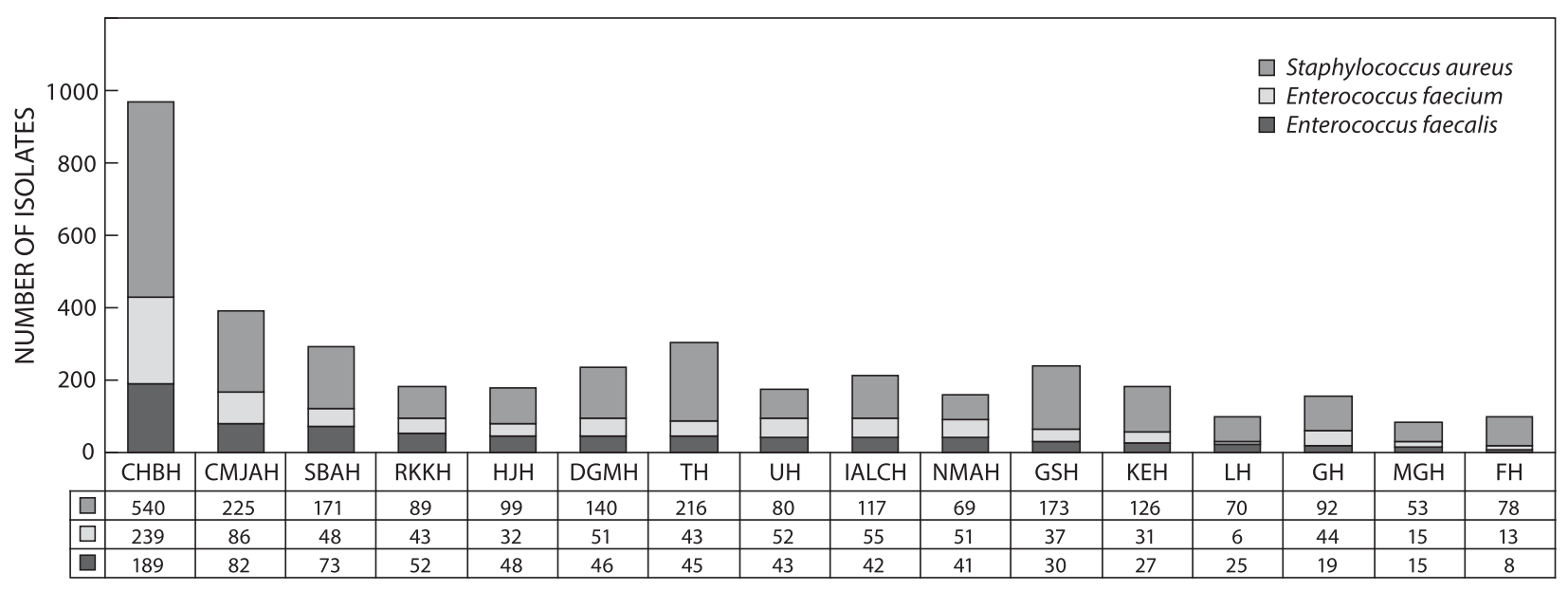

Figure 5: Number of Gram-positive bacteria: Enterococcus faecalis $(n=785)$, Enterococcus faecium $(n=846)$ and Staphylococcus aureus ( $n=2$ 338) reported from 16 sentinel hospitals across South Africa, January 1, 2016 to December 31, 2016.

Table 9: Antimicrobial susceptibility patterns of Gram-positive bacteria isolated from blood cultures reported from 16 sentinel hospitals across South Africa, January 1, 2016 to December 31, 2016

\begin{tabular}{|c|c|c|c|c|c|c|c|c|c|c|c|c|}
\hline \multirow{3}{*}{$\begin{array}{l}\text { Antimicrobial } \\
\text { agent }\end{array}$} & \multicolumn{4}{|c|}{ Enterococcus faecalis } & \multicolumn{4}{|c|}{ Enterococcus faecium } & \multicolumn{4}{|c|}{ Staphylococcus aureus } \\
\hline & \multicolumn{2}{|c|}{ Non-susceptible } & \multicolumn{2}{|c|}{ Susceptible } & \multicolumn{2}{|c|}{ Non-susceptible } & \multicolumn{2}{|c|}{ Susceptible } & \multicolumn{2}{|c|}{ Non-susceptible } & \multicolumn{2}{|c|}{ Susceptible } \\
\hline & $n$ & $\%$ & $n$ & $\%$ & $n$ & $\%$ & $n$ & $\%$ & n & $\%$ & $n$ & $\%$ \\
\hline Linezolid & 3 & 0.4 & 687 & 99.6 & 5 & 0.7 & 734 & 99.3 & - & - & - & - \\
\hline $\begin{array}{l}\text { Penicillin/ } \\
\text { ampicillin }\end{array}$ & 33 & 9.7 & 306 & 90.3 & 383 & 97.5 & 10 & 2.5 & - & - & - & - \\
\hline Teicoplanin & 5 & 1.2 & 409 & 98.8 & 12 & 2.7 & 426 & 97.3 & - & - & - & - \\
\hline Vancomycin & 8 & 1.0 & 759 & 99.0 & 45 & 5.4 & 796 & 94.6 & - & - & - & - \\
\hline Cloxacillin & - & - & - & - & - & - & - & - & 709 & 30.8 & 1590 & 69.2 \\
\hline
\end{tabular}

Notes: number of isolates $(n)$, percentage $(\%)$, not reported (-).

Vancomycin was not reported for Staphylococcus aureus as non-susceptibility is rare.

Enterococcus faecium and 59\% (2 338/3 369) were identified as Staphylococcus aureus. All three pathogens were reported from all 16 sentinel hospitals in South Africa. Approximately 29\% (968/3 369) of all three pathogens were reported from Chris Hani Baragwanath Hospital (Figure 5).

Of the panel of antimicrobial agents that were tested, more than $90 \%$ of Enterococcus faecalis and Enterococcus faecium isolates were shown to be susceptible to oxazolidinones and glycopeptides (Table 9). In comparison with 2015, AST patterns for the particular antimicrobial agents remained similar in both Enterococcus faecalis and Enterococcus faecium isolates over the two-year period (Figure 6). There were no unusual AST patterns reported for Enterococcus faecalis isolates (Table 10). Approximately $48 \%$ of Enterococcus faecium isolates from Universitas Hospital were shown to be non-susceptible to

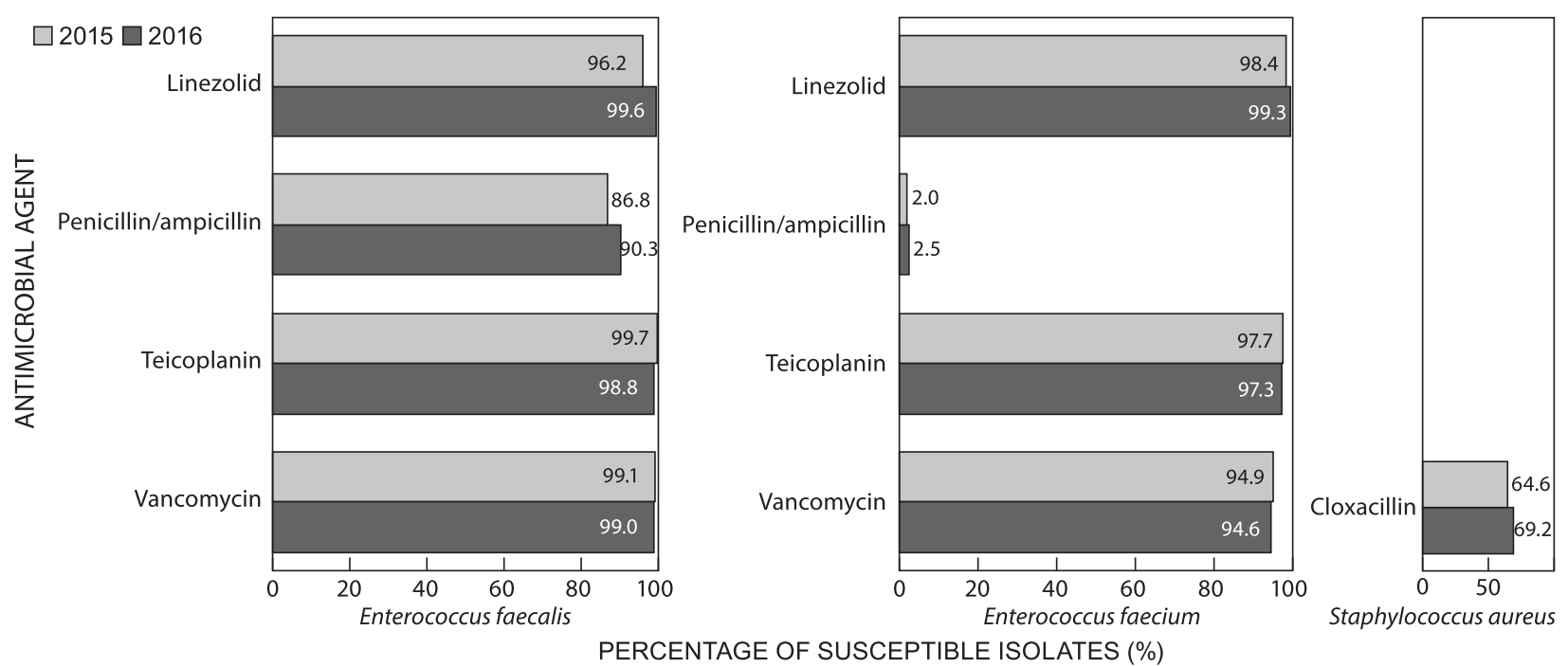

Figure 6: Percentage of susceptible Enterococcus faecalis, Enterococcus faecium and Staphylococcus aureus isolates, 2015 to 2016. 
Table 10: Number and percentage of susceptible Enterococcus faecalis isolates per antimicrobial agent from 16 sentinel hospitals across South Africa, January 1, 2016 to December 31,2016

\begin{tabular}{|c|c|c|c|c|c|c|c|c|c|c|c|c|c|c|c|c|}
\hline $\begin{array}{l}\text { Antimicrobial } \\
\text { agent }\end{array}$ & CHBH & СMJAH & DGMH & SBAH & GSH & TH & $\mathrm{HJH}$ & KEH & IALCH & $\mathrm{UH}$ & GH & FH & NMAH & LH & RKKH & MGH \\
\hline & $\bar{n}$ & $\bar{n}$ & $n$ & $n$ & $n$ & $\bar{n}$ & $n$ & $\bar{n}$ & $n$ & $n$ & $n$ & $n$ & $n$ & $n$ & $n$ & $n$ \\
\hline & $\%$ & $\%$ & $\%$ & $\%$ & $\%$ & $\%$ & $\%$ & $\%$ & $\%$ & $\%$ & $\%$ & $\%$ & $\%$ & $\%$ & $\%$ & $\%$ \\
\hline \multirow[t]{2}{*}{ Linezolid } & 188 & 79 & 43 & 73 & - & 40 & 45 & - & 40 & 43 & - & - & - & - & 36 & - \\
\hline & 100.0 & 100.0 & 97.7 & 100.0 & - & 100.0 & 97.8 & - & 100.0 & 100.0 & - & - & - & - & 100.0 & - \\
\hline \multirow{2}{*}{$\begin{array}{l}\text { Penicillin/ } \\
\text { ampicillin }\end{array}$} & - & 62 & - & 69 & - & - & - & - & 39 & - & - & - & - & - & 46 & - \\
\hline & - & 91.9 & - & 98.6 & - & - & - & - & 100.0 & - & - & - & - & - & 91.3 & - \\
\hline \multirow[t]{2}{*}{ Teicoplanin } & - & 64 & 41 & 72 & - & 42 & - & - & 40 & - & - & - & - & - & 38 & - \\
\hline & - & 100.0 & 97.6 & 100.0 & - & 100.0 & - & - & 100.0 & - & - & - & - & - & 97.4 & - \\
\hline \multirow[t]{2}{*}{ Vancomycin } & 189 & 79 & 43 & 72 & - & 45 & 43 & - & 42 & 43 & - & - & 39 & - & 53 & - \\
\hline & 99.5 & 100.0 & 100.0 & 100.0 & - & 100.0 & 100.0 & - & 100.0 & 95.3 & - & - & 94.9 & - & 96.2 & - \\
\hline
\end{tabular}

Note: Data were omitted for those sentinel hospitals that tested fewer than 30 ESKAPE pathogens for a particular antimicrobial agent.

Table 11: Number and percentage of susceptible Enterococcus faecium isolates per antimicrobial agent from 16 sentinel hospitals across South Africa, January 1, 2016 to December 31,2016

\begin{tabular}{|c|c|c|c|c|c|c|c|c|c|c|c|c|c|c|c|c|}
\hline $\begin{array}{l}\text { Antimicrobial } \\
\text { agent }\end{array}$ & CHBH & CMJAH & DGMH & SBAH & GSH & TH & $\mathrm{HJH}$ & KEH & IALCH & UH & GH & FH & NMAH & LH & RKKH & MGH \\
\hline & $n$ & $n$ & $n$ & $n$ & $n$ & $n$ & $n$ & $n$ & $n$ & $n$ & $n$ & $n$ & $n$ & $n$ & $n$ & $n$ \\
\hline & $\%$ & $\%$ & $\%$ & $\%$ & $\%$ & $\%$ & $\%$ & $\%$ & $\%$ & $\%$ & $\%$ & $\%$ & $\%$ & $\%$ & $\%$ & $\%$ \\
\hline \multirow[t]{2}{*}{ Linezolid } & 232 & 84 & 51 & 43 & 37 & 39 & 32 & - & 49 & 52 & 39 & - & - & - & - & - \\
\hline & 100.0 & 98.8 & 94.1 & 100.0 & 100 & 97.4 & 100.0 & - & 100.0 & 100.0 & 100.0 & - & - & - & - & - \\
\hline \multirow{2}{*}{$\begin{array}{l}\text { Penicillin/ } \\
\text { ampicillin }\end{array}$} & - & 79 & - & 43 & 37 & - & - & - & 53 & - & 41 & - & - & - & 38 & - \\
\hline & - & 2.5 & - & 2.3 & 0.0 & - & - & - & 1.9 & - & 7.3 & - & - & - & 2.6 & - \\
\hline \multirow[t]{2}{*}{ Teicoplanin } & - & 81 & 47 & 45 & 37 & 40 & - & 30 & 52 & - & 39 & - & - & - & - & - \\
\hline & - & 96.3 & 93.6 & 97.8 & 100.0 & 100.0 & - & 100.0 & 98.1 & - & 100.0 & - & - & - & - & - \\
\hline \multirow[t]{2}{*}{ Vancomycin } & 236 & 87 & 51 & 46 & 37 & 44 & 30 & 31 & 55 & 52 & 44 & - & 51 & - & 43 & - \\
\hline & 96.2 & 95.4 & 96.1 & 97.8 & 100.0 & 100.0 & 100.0 & 100.0 & 98.2 & 51.9 & 100.0 & - & 96.1 & - & 97.7 & - \\
\hline
\end{tabular}




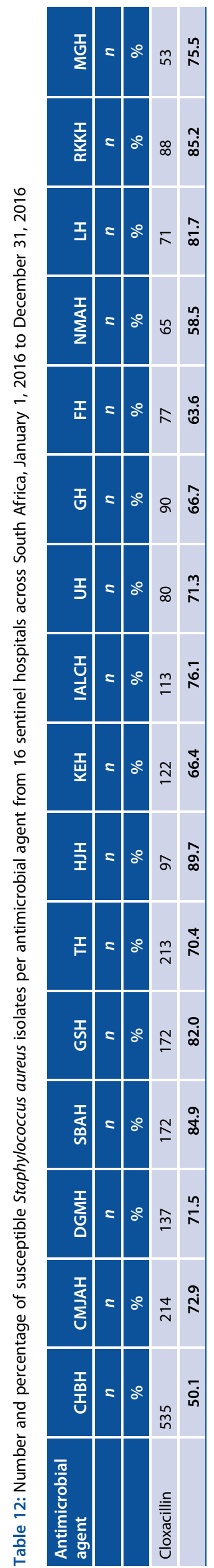

vancomycin; however, this finding should be interpreted with caution as AST testing for these non-susceptible isolates may not have been confirmed using additional testing (Table 11).

Approximately $69 \%$ of Staphylococcus aureus isolates were susceptible to cloxacillin (Table 9). In comparison with 2015, susceptibility to cloxacillin $(p=0.23)$ increased from $65 \%$ to $69 \%$; however, this was not statistically significant (Figure 6). In addition, 50\% of Staphylococcus aureus isolates reported from Chris Hani Baragwanath Hospital were shown to be non-susceptible to cloxacillin (Table 12).

\section{Carbapenemase-producing Enterobacteriaceae}

In 2016, AMRL/CHARM identified 1182 CPE isolates from clinically significant sites (blood and urine were the most common specimen types). Approximately $72 \%$ (846/1 182) of CPE isolates were identified as Klebsiella pneumoniae. Approximately $34 \%(400 / 1182)$ and $63 \%(741 / 1182)$ of CPE isolates were shown to be positive for $b / a_{\mathrm{NDM}-1}$ and $b / a_{\mathrm{OXA}-48}$-like encoding genes (Table 13). In 2016, CPE isolates encoding for bla $a_{\mathrm{OXA}}$ ${ }_{48}$ like genes were shown to be most prevalent compared with $2015 .^{6}$

\section{Limitations}

\section{Interpretation of results}

The results of this report should be interpreted with caution. A number of factors might have introduced bias, resulting in either an overestimation or underestimation of AST reporting.

1. Data may have been incomplete due to missing cases not captured on the LIS or non-standardised coding of ESKAPE pathogens and antimicrobial agents at diagnostic laboratories. Testing methods and microbiological practice may have varied between sentinel hospitals and this could account for variations in the results presented in this report.

2. Confirmatory AST methods may not have been performed or recorded for any of these ESKAPE pathogens as the results presented here were reported as captured on the LIS by diagnostic laboratories. We have not been able to report on colistin AST as new methods have been recommended by CLSI and the European Committee on Antimicrobial Susceptibility Testing (EUCAST) guidelines, which have not yet been implemented by diagnostic laboratories.

3. For some sentinel hospitals, ESKAPE pathogens may not all have been represented. This may be due to ESKAPE pathogens not being isolated at a particular sentinel hospital in 2016.

4. Data were omitted for those sentinel hospitals that tested fewer than 30 ESKAPE pathogens for a particular antimicrobial agent.

5. Vancomycin resistance for Staphylococcus aureus requires confirmatory testing, which may not have been available at routine laboratory level. All Staphylococcus aureus isolates that are non-susceptible to vancomycin should be referred to AMRL/CHARM at the NICD.

6. Results for CPE may not be representative as not all CRE isolates are referred to CHARM for CPE confirmatory testing. 
Table 13: Total number of confirmed Carbapenemase-producing Enterobacteriaceae, January 1, 2016 to December 31, 2016

\begin{tabular}{|c|c|c|c|c|c|c|c|}
\hline \multirow[b]{2}{*}{ CPE } & \multicolumn{6}{|c|}{ Carbapenemase class } & \multirow[b]{2}{*}{ Total } \\
\hline & GES & IMP & KPC & $\begin{array}{l}\text { OXA-48 and } \\
\text { variants }\end{array}$ & NDM & VIM & \\
\hline $\begin{array}{l}\text { Citrobacter } \\
\text { amalonaticus }\end{array}$ & - & - & - & 2 & - & - & 2 \\
\hline $\begin{array}{l}\text { Citrobacter } \\
\text { braakii }\end{array}$ & - & - & - & 1 & 1 & - & 2 \\
\hline $\begin{array}{l}\text { Citrobacter } \\
\text { freundii }\end{array}$ & - & - & 1 & 8 & 9 & - & 18 \\
\hline Citrobacter koseri & - & - & - & 1 & - & - & 1 \\
\hline $\begin{array}{l}\text { Citrobacter } \\
\text { sedlakii }\end{array}$ & - & - & - & - & 1 & - & 1 \\
\hline $\begin{array}{l}\text { Enterobacter } \\
\text { aerogenes }\end{array}$ & - & - & - & 8 & 1 & - & 9 \\
\hline $\begin{array}{l}\text { Enterobacter } \\
\text { cloacae }\end{array}$ & 1 & - & 2 & 57 & 32 & 2 & 94 \\
\hline $\begin{array}{l}\text { Enterobacter } \\
\text { gergoviae }\end{array}$ & - & - & - & 1 & - & - & 1 \\
\hline $\begin{array}{l}\text { Enterobacter } \\
\text { kobei }\end{array}$ & - & - & - & 1 & 2 & - & 3 \\
\hline Escherichia coli & - & - & - & 90 & 11 & - & 101 \\
\hline Klebsiella oxytoca & 1 & - & - & 6 & 2 & - & 9 \\
\hline $\begin{array}{l}\text { Klebsiella } \\
\text { pneumoniae }\end{array}$ & 11 & - & 3 & 531 & 287 & 14 & 846 \\
\hline Klebsiella species & - & - & - & 6 & - & 2 & 8 \\
\hline $\begin{array}{l}\text { Morganella } \\
\text { morganii }\end{array}$ & - & - & - & 2 & 6 & - & 8 \\
\hline Proteus mirabilis & - & - & - & 2 & - & - & 2 \\
\hline Proteus vulgaris & - & - & - & - & 1 & - & 1 \\
\hline $\begin{array}{l}\text { Providencia } \\
\text { rettgeri }\end{array}$ & - & - & - & 1 & 17 & - & 18 \\
\hline $\begin{array}{l}\text { Salmonella } \\
\text { species }\end{array}$ & - & - & - & - & 1 & - & 1 \\
\hline $\begin{array}{l}\text { Serratia } \\
\text { marcescens }\end{array}$ & 3 & - & - & 24 & 29 & 1 & 57 \\
\hline Total & 16 & 0 & 6 & 741 & 400 & 19 & 1182 \\
\hline
\end{tabular}

Notes: imipenemase (IMP), Guiana extended-spectrum carbapenemase (GES) Klebsiella pneumoniae carbapenemase (KPC), oxacillinase (OXA), New Delhi metallo-beta-lactamase (NDM) and veronica integron metallo-beta-lactamases types (VIM).

\section{Conclusion}

In this report, data showed that antimicrobial susceptibility patterns for Klebsiella pneumoniae remained the same over the two-year period. Antimicrobial resistance to third and fourth generation cephalosporins increased for Escherichia coli. Carbapenem resistance in Acinetobacter baumannii is of concern as there are limited antimicrobial options available for treatment of significant infections. Although a large proportion of vancomycin-resistant Enterococcus faecium was reported from Universitas Hospital, these isolates need laboratory confirmation as this may have been an unidentified outbreak. In most pathogens, the AST patterns remained unchanged. There has been a large increase in the number of CPEs identified across South Africa over the two-year period. Enhanced surveillance together with effective antimicrobial stewardship programmes and strict infection control practices are needed to combat AMR in both ESKAPE pathogens and CPEs. The limitations highlighted in this report emphasise the need for continuous improvement in quality of data obtained by electronic surveillance.

\section{Disclaimer}

Data are reported as received through the CDW. No demographic, epidemiological, clinical or molecular data were available to distinguish between hospital-associated and community-associated infections.

Acknowledgements - The authors would like to thank the following: Ms Sue Candy and her team for preparing the data; $\mathrm{Dr}$ Ashika Singh-Moodley for providing 2016 CPE data; the SASCM editorial committee (Prof. O Perovic, Dr W Lowman, Prof. N Govender, Dr C Sriruttan, Dr K Moodley, Dr C Govind, Dr I Zietsman, Dr B Magazi, Dr R Kularatne, Dr M Maloba, Dr C Bamford, Dr K Sweswe-Han and Dr Y Mahabeer) for comments and suggestions.

Disclosure statement - No potential conflict of interest was reported by the authors. 


\section{References}

1. De Rosa FG, Corcione $S$, Pagani N, et al. From ESKAPE to ESCAPE, from KPC to CCC. CID. 2015;60(8): 1289-1290. 10.1093/cid/ciu1170

2. Dik JH, Sinha B. Challenges for a sustainable financial foundation for antimicrobial stewardship. Infect Dis Rep. 2017;9: 32-34.

3. Patel JB, Cockerill FR, Eliopoulos GM, et al. CLSI. Performance Standards for Antimicrobial Susceptibility Testing. 26th ed. CLSI supplement M100S. 2016;36(1): 1-12. Wayne, PA: Clinical and Laboratory Standards Institute.

4. Bamford C, Brink A, Govender N, et al. Part V. Surveillance activities. SAMJ. 2011;101: 1-8.

5. Performance Standards for Antimicrobial Susceptibility Testing Clinical and Laboratory Standards Institute (CLSI), 2016; M100S26.

6. Perovic O, Chetty V [Internet]. Antimicrobial Resistance Surveillance from sentinel public hospitals, South Africa. 2015 [Updated August 2016; cited 05 July 2017]. Available from: http:// www.fidssa.co.za/Content/images/2015_SASCM_Public_Sector_ ReportFINAL.pdf 